\title{
Éditorial
}

\section{Comme le rofecoxib, la codéine sera-t-elle retirée du commerce?}

\section{Rofecoxib out of production : codeine will be the next?}

Hugo NEELS, Sarah MAHIEU

ZNA Stuivenberg, Laboratoire de Toxicologie, Lange Beeldekenstraat, 267 - B-2060 ANTWERPEN - BELGIQUE

Tél : +32 (0)32 177804 - Mél : hugo.neels@zna.be

Le rofecoxib (Vioxx®i) a été retiré du commerce, car estimé dangereux pour les patients cardiaques. Si on peut en croire le «brief-report» du New England Journal of Medicine (1), la codéine devrait également faire l'objet d'une discussion critique.

La codéine est en réalité une prodrogue de la morphine (2). Environ $10 \%$ de la codéine est bio-activée en morphine par une monooxygénase à cytochrome $\mathrm{P} 450$ (CYP2D6, O-déméthylation). La codéine est déméthylée pour former la norcodéine qui est inactive et glucuroconjuguée en codéine-6-glucuronide, également inactive. La biotransformation de la codéine est soumise à un polymorphisme génétique important. En effet, 1 à $7 \%$ des caucasiens et plus de $25 \%$ des Ethiopiens ont des duplications de gènes et sont considérés comme métaboliseurs ultrarapides; des personnes avec 2 allèles fonctionnel normaux sont des métaboliseurs extensifs. Enfin, pour 7 à $10 \%$ de la population qui n'a pas d'allèles fonctionnels, la codéine est sans effet (3). Cet article (1) décrit le sort d'une personne de 62 ans avec une histoire de leucémie chronique lymphocytaire, qui se plaignait de fatigue depuis 3 jours, de respiration difficile, de fièvre et de toux. Sa dernière chimiothérapie datait de 3 mois. Le patient recevait uniquement de façon quotidienne $1500 \mathrm{mg}$ d'acide val- proïque pour un traitement d'épilepsie grand mal post traumatique. Lors de son entrée à l'hôpital le patient était hypoxémique (les pressions partielles en oxygène artériel et en dioxyde de carbone étaient respectivement de 56 et $37 \mathrm{~mm} \mathrm{Hg}$ ) (intervalle de référence : $\mathrm{O}_{2}: 80$ $100 \mathrm{~mm} \mathrm{Hg}, \mathrm{CO}_{2}: 35-45 \mathrm{~mm} \mathrm{Hg}$ ). D'autres analyses ont conduit au diagnostic d'une pneumonie bilatérale causée par une mycose (statut immunologique compromis). Le traitement a consisté en ceftriaxone, claritromycine et voriconazol. Pour apaiser la toux, il avait été prescrit $3 \times 25 \mathrm{mg}$ de codéine par jour. Le quatrième jour de l'hospitalisation, la situation du patient s'est aggravée rapidement, sa pression partielle d'oxygène est montée à $80 \mathrm{~mm} \mathrm{Hg}$ avec une pCO2 restée au même niveau. Le patient fut traité par ventilation assistée et fut transféré vers une unité de soins intensifs. Quatre vingt dix minutes plus tard, la pO2 était à $68 \mathrm{mmHg}$ mais le coma, mesuré avec le Coma Glasgow Scale, restait le même. Dans le sérum, l'urée et la créatinine avaient légèrement augmenté mais l'hyperhydratation a permis de normaliser ces paramètres. La situation du patient s'est améliorée de façon significative avec une injection IV de $400 \mu \mathrm{g}$ de naloxone. Une perfusion continue $(0,4 \mathrm{mg}$ naloxone par heure pendant 6 heures) a eu pour effet le retour à une conscience normale et la 
disparition de l'insuffisance respiratoire. Après 48 heures, l'état du patient s'était entièrement normalisé.

Le génotypage du CYP2D6 a révélé la présence d'au moins 3 allèles fonctionnels. Le phénotypage du CYP2D6 (administration per os de $25 \mathrm{mg}$ de dextrométorphan déméthylé en dextrorphan) ne permettait plus de mettre en évidence aucune trace de dextrométorphan dans les urines recueillies après 8 heures. Le rapport dextromethorphan/dextrorphan était inférieur à 0,005 ce qui est compatible avec un métabolisme ultrarapide.

On peut se poser la question de ce qui est arrivé à ce patient. Plusieurs raisons peuvent expliquer la dépression respiratoire survenue dans ce cas : (i) le métabolisme utrarapide a déclenché une transformation excessive de codéine en morphine : plus de catalisateur signifie plus de morphine. La concentration plasmatique de morphine était de $80 \mu \mathrm{g} / \mathrm{L}$ pour une valeur attendue de 1 à $4 \mu \mathrm{g} / \mathrm{L}$ ! (ii). L'interaction médicamenteuse (inhibition du CYP3A4 par le voriconazole et la claritromycine) a aggravé l'accumulation de la morphine.. Enfin, la situation transitoire d'insuffisance rénale a eu pour effet l'accumulation de la morphine-6-glucuronide, dont on sait qu'elle a non seulement plus d'effets analgésiques que la morphine, mais également plus d'effets indésirables.

\section{Références}

1. Gasche Y., Daali Y., Fathi M., Chiappe A., Cottini S., Dayer P., Desmeules J. Codeine intoxication associated with ultrarapid CYP2D6 metabolism. N. Engl. J. Med. 2004 ; 351 : 2827-31.

2. Desmeules J, Gascon M.P., Dayer P., Magistris M. Impact of environmental and genetic factors on codeine analgesia. Eur. J. Clin. Pharmacol. 1991 ; 41 : 23-6.

3. Sindrup S.H., Brosen $\mathrm{K}$. The pharmacogenetics of codeine hypoalgesia. Pharmacogenetics $1995 ; 5$ : 335-46. 